Article

\title{
Determination of Project Procurement Method with a Graphical Analytic Model
}

\author{
Kwo-Wuu Wang ${ }^{1}$, Yuan-Yu Hsu ${ }^{2}$, Wen-der Yu ${ }^{3, *}$ and Shao-tsai Cheng ${ }^{4}$ \\ 1 Management College, National Defense University, Taoyuan City 33449, Taiwan; wangkwo4@yahoo.com.tw \\ 2 Department of Civil Engineering, Chung Hua University, Hsinchu City 30012, Taiwan; \\ yu5053tw@yahoo.com.tw \\ 3 Department of Construction Engineering, Chaoyang University of Technology, Taichung City 41349, Taiwan \\ 4 Department of Construction Management, Chung Hua University, Hsinchu City 30012, Taiwan; \\ shaotsai@chu.edu.tw \\ * Correspondence: wenderyu@cyut.edu.tw
}

Received: 17 August 2018; Accepted: 2 October 2018; Published: 8 October 2018

\begin{abstract}
Procurement is a key stage affecting the successful delivery of a sustainable project. Previous project procurement practice employed either the Lowest Tender (LT) method for homogeneous projects or the Best Value (BV) method for heterogeneous projects. Recently, a new type of procurement method for moderately heterogeneous project, the Heterogeneous Lowest Tender (HLT), was introduced and it has proven to be more beneficial and sustainable to clients in terms of both cost-effectiveness and the delivery performance of project products/results. However, statistics also showed that only $0.33 \%$ of public works adopt HLT for project procurement in Taiwan due to the lack of a useful method selection model. To solve this problem, this paper proposes an enhanced graphical method that is based on a previously defined Price Elasticity of Performance (PEP) measure, namely the Graphical Analysis Method for Price Elasticity of Performance (GAM-PEP), to select a more appropriate method from among the three prevailing project procurement methods: LT, BV, and HLT, so that the sustainability objectives of a project can be better realized. Finally, a real-world case construction procurement project was selected to demonstrate the feasibility of the proposed model. It is concluded that the proposed GAM-PEP outperforms the previous models in determining the most sustainable project procurement method.
\end{abstract}

Keywords: best value; lowest tender; project sustainability; heterogeneous procurement

\section{Introduction}

Procurement is one of the most important stages in construction project management; its primary purpose is to determine the most appropriate contractor to successfully deliver the project. According to the Government Procurement Agreement of the World Trade Organization (WTO) [1] and the Government Procurement Act (GPA) of Taiwan [2], there are two prevailing contractor selection methods that are widely adopted for project procurement: (1) the Lowest Tender (LT), which is based on selecting the lowest bid from among the submitted tenders; and, (2) Best Value (BV), selecting the contractor who is the most advantageous to the client, in terms of price, as well as performance factors, including economic, social, and environmental sustainability objective [3]. Traditionally, the LT is more widely adopted than BV [4], since it selects the most favorable contractor with price as the primary concern; whichever contractor offers the lowest price (usually below the agency's budget) wins the tender [5]. On the other hand, the BV is a procurement method that looks at factors other than price (e.g., quality, expertise, and sustainability) when selecting the contractor. Using the BV method, the bidder considers not only the price, but also the performance factors, in order to maintain 
a long-term relationship with the procurement agency [6], since only the contractor who provides the highest values (or the best performance) to the client wins the tender [7]. As a result, BV has shifted the paradigm for competitive bidding from traditional competition over price to competition over heterogeneous value [8]. With such a perspective, BV is more useful in selecting a more appropriate contractor to achieve the sustainability objectives of a project.

After seven years of the practical implementation of the BV method in Taiwan, it was found that a gap exists between BV and LT for procurement projects that are neither sufficiently heterogeneous for $\mathrm{BV}$, nor apparently homogeneous. As a result, a new type of contractor selection method for heterogeneous procurement, the Heterogeneous Lowest Tender (HLT) [9], has been introduced to fill in the gap between BV and LT. The procedure of HLT is similar to that of BV procurement. It starts with organizing an Expert Evaluation Committee (EEC) to determine the criteria for evaluating the heterogeneity of the tenders and the lowest acceptable performance score (LAP) for the procurement. After receiving the submitted tenders, the members of EEC are called to evaluate the tenders to determine their associated performance scores according to the pre-determined criteria. The tenders with performance scores higher than the LAP will enter the second phase for competitive bidding, in which the tender with the lowest price will win the priority for contract negotiation (PCN). Should the contract negotiation reach a consensus between the bidder and the client, the bidder with PCN will be awarded the contract.

Some researchers have found that HLT combines the merits of both BV and LT by awarding the tender to the bidder who offers the lowest price from among those who are pre-qualified via a committee evaluation process $[10,11]$. According to Su's empirical study, the performance of the delivered projects procured via HLT has proven to be similar to that of BV [10]. Moreover, based on a wide range survey conducted by Chen [10] on 59,126 historical public procurement projects of the Taiwan Government during 2002 2007, it was found that the average percentage of the 'Awarded Price over the Budget $(\mathrm{APOB} \%)^{\prime}$ ratio $(\mathrm{APOB} \%=($ Awarded Price $\div$ Budget $) \times 100 \%)$ for the three contractor selection methods were: (1) $91.64 \%$ for LT procurement projects; (2) $99.27 \%$ for BV procurement projects; and, (3) $89.84 \%$ for HLT procurement projects. Since APOB $\%$ is a commonly used indicator for determining the cost-effectiveness of project procurement, such results revealed that HLT not only delivers high performance/quality projects like the BV does, but it is also as cost-effective as the LT.

Although the HLT outperforms other procurement methods both in performance and cost-effectiveness, Chen's survey [10] also showed that only $0.33 \%$ of the 59,126 historical procurement cases (accounting for 195 projects) were selected via HLT; 3.17\% (accounting for 1874 projects) were selected via BV; and the rest, the majority of $96.50 \%$ (accounting for 57,057 projects) were procured via the traditional LT method. It is obvious that, although they are more appropriate for the procurement of sustainable projects, the heterogeneous procurement methods (including BV and HLT) are not very popular with the construction practitioners in Taiwan or in other countries. It is believed that the lack of an effective tool for the project owners' procurement personnel to determine the most appropriate procurement method is the primary reason hindering the procurement staffs from adopting innovative contractor selection methods [12].

Although Wang and Yu [13] developed an analytic geometrical approach (namely the Price/Performance Analysis Model, P/PAM) to select between the LT and the BV procurement schemes, no analytic approach is yet available to determine the HLT method choice. Nevertheless, the P/PAM method of Wang and Yu [13] requires complicated mathematical calculation and a tedious process to determine the market heterogeneity, and it is thus difficult for construction practitioners to implement. As a result, a simplified method without complicated mathematical calculations, and a more powerful model to differentiate from BV and HLT is desired. This paper aims at developing a graphical analytic method, namely the Graphical Analysis Method for Price Elasticity of Performance (GAM-PEP), to address the gap that exists in selecting a more appropriate project procurement method among the LT, BV, and HLT. 


\section{Review of Related Works}

\subsection{Definition of Heterogeneity}

According to the requirements of GPA, the appropriate procurement method for a procurement project should be determined according to the project's heterogeneity [2]. Highly heterogeneous projects must be procured by using the BV method, while less heterogeneous projects should be procured while using the LT method. Projects with medium heterogeneity should be procured using the HLT method. However, the 'heterogeneity' defined in the GPA is neither quantitative nor sufficiently operational. It is difficult for the project owners' procurement personnel to make appropriate decisions when determining the most appropriate procurement method. As a result, the procurement personnel tend to avoid adopting BV or other heterogeneous procurement methods in order not to violate the law [13]. To attack such a drawback, Yu and Wang [12] proposed the "Price Elasticity of Performance (PEP)" as a quantitative measurement for the 'heterogeneity' of a procurement project. The PEP is denoted as " $E$ " hereafter and was defined by Yu and Wang [12], as follows:

$$
E=\frac{\frac{\Delta Q}{Q}}{\frac{\Delta P}{P}}=\frac{\Delta Q}{\Delta P} \times \frac{P}{Q}=\frac{1}{m} \times \frac{P}{Q}
$$

where, $E$ is the measurement of PEP, $Q$ is the measure of work performance, $\frac{\Delta Q}{Q}$ is the percentage of change in work performance, $P$ is the price, $\frac{\Delta P}{P}$ is the percentage of change in the price, and $m$ is the slope of the tangent at point $(Q, P)$ in the Q-P Diagram, as described in the next section.

When the range of performance that is provided by different bidders is relatively wide, a slight increase in the price encourages the bidder to significantly improve the performance, e.g., $\frac{\Delta Q}{Q}>\frac{\Delta P}{P}$ $(E>1)$. Therefore, a higher value of $E$ implies more heterogeneous bidders. Conversely, as $\frac{\Delta Q}{Q}<\frac{\Delta P}{P}$, the $E$ is $<1$. Therefore, a lower value of $E$ implies that the bidders in the market are less heterogeneous.

If all contractors bidding for the same project are considered as a "market", the collective

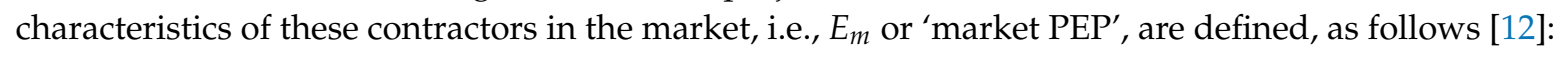

$$
E_{m}=\sum_{i=1}^{m} k_{i} E_{i}
$$

where $E_{m}$ is the index of the 'market $\mathrm{PEP}^{\prime}, m$ is the total number of bidders, $k_{i}$ is the weighting of the $i$ th bidder in the market, which is defined in Equation (3), and $E_{i}$ is the performance elasticity of the $i$ th bidder in the market.

In Equation (2), $k_{i}$ is calculated, as follows:

$$
k_{i}=\frac{q_{i}}{Q}
$$

where $q_{i}$ is the value of the multi-criteria Performance Index (PI) for the $i$ th bidder and $Q$ is the summation of the values of the performance index for all bidders in the market.

\subsection{Measuring the Price Elasticity of Performance (PEP)}

To measure the PEP for a tender offered by a bidder, the point elasticity of the tender must be calculated. Wang and $\mathrm{Yu}$ [13] suggest an analytic geometrical approach (namely Price/Performance Analysis Model, P/PAM) to determine the PEP for a tender, namely a Performance/Price diagram (Q-P Diagram) using the original concept of the point price elasticity for demand that was proposed by Marshall [14] (see Figure 1). 


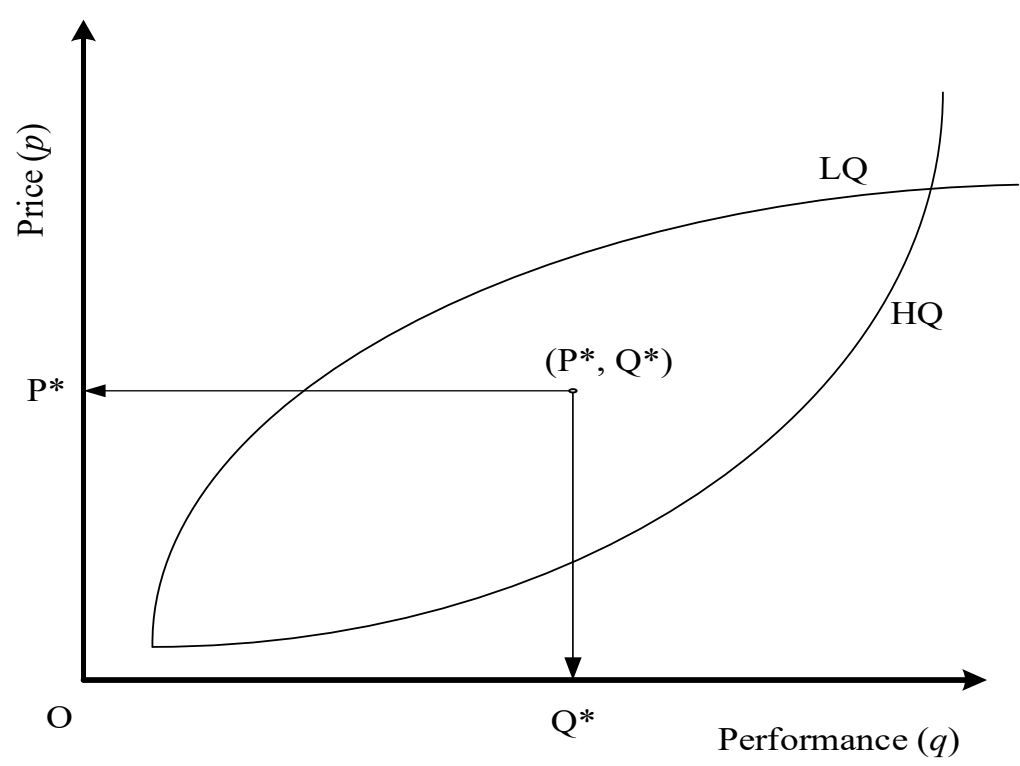

Figure 1. The Performance/Price diagram (Q-P Diagram) (redrawn from [12]).

Figure 1 shows the relationship between the price and the multi-criteria performance factors in terms of technology, quality, function, benefit, characteristic, or other business aspects for a tender on a two-dimensional (2-D) diagram. The $X$-axis represents the broadly defined 'performance' (denoted as ' $Q$ '), and the $Y$-axis represents the 'price' (denoted as ' $P$ ') for the product/work offered by the bidder. From the viewpoint of the bidder, a specific point $\left(Q^{*}, P^{*}\right)$ on the Q-P Diagram represents the performance level $\left(Q^{*}\right)$ and the associated bid price $\left(P^{*}\right)$ offered by the bidder. There are two envelope curves of interest in the Q-P Diagram: (1) LQ, the lowest-quality curve, which links all of the lowest-quality bidders at the same bid-price level and (2) HQ, the highest-quality curve, which links all of the highest-quality bidders at the same bid-price level. According to Dolan and Simon [15], the bid-price level increases as the quality of the product improves. In other words, a product with better performance is quoted at a higher price. However, the marginal cost for the improved performance increases dramatically beyond a certain level of performance due to the technological capability limitations of the contractors, resulting in a concave HQ curve. Similarly, the client's conceived value (i.e., the price that the client is willing to pay) increases when the product performance improves. However, the marginal utility of the performance of the client is decreased when the price increases because of the limited budget, resulting in a convex LQ curve.

The P/PAM method provides a useful tool for the selection between the LT and BV schemes; however, it is unable to differentiate between the BV and HLT. Moreover, the construction of the $\mathrm{P} / \mathrm{PAM}$ model requires complicated mathematical calculations. It is not easy to implement for the construction practitioners.

\subsection{Measuring Project Sustainability for Heterogeneous Procurement}

Achieving project sustainability has attracted the attention of researchers since the early 1990s. There are three widely adopted pillars for project sustainability: economic, social, and environmental [3]. In order to assess the sustainability of a project, several researchers have developed different project-wise assessment frameworks [16-18]. Other researchers in the field of construction have attempted various approaches to improve the sustainability of a project, including green innovation of construction methods [19,20], promotion of green building technologies [21], and the implementation of techniques and initiatives for eliminating GHG emissions [22,23].

In this paper, a recently developed project lifecycle sustainability assessment method called the Construction Project Sustainability Assessing System (CPSAS) [24] is adopted for measuring the sustainability of the project execution plan proposed by the bidder during the procurement stage. 
The framework of CPSAS comprises four levels: (1) Level-1: Sustainability Pillars (SP), whose 3 pillars of sustainability include environmental sustainability (E), social sustainability (S), and economic sustainability (EC); (2) Level-2: Sustainability Categories (SC), whose eight sustainability categories belonging to the three pillars are defined; (3) Level-3: Sustainability Sub-Categories (Sub-SC), whose 19 sustainability sub-categories are identified for the eight sustainability categories; and, (4) Level-4: Sustainability Indicators (SI), whose 31 sustainability indicators are identified. Detailed definitions of the sustainability indicators can be found in Yu et al. [24].

The CPSAS assesses project sustainability using different sets of Sustainability Indicators (SIs) in the stages of a project lifecycle with different levels of significance. For example, for the Construction stage, 13 SIs are considered 'Very important', 10 SIs are 'Important', and the other eight SIs are either of 'Medium' or 'Minor' importance. Table 1 shows the suggested SIs for assessment in the Construction stage by CPSAS [24]. The fourth column (Criterion) of Table 1 indicates the criteria values for determining whether the associated Sustainability Indicators are considered 'sustainable'. If the values of the associated SIs satisfy the requirement value, then they are considered as 'sustainable'; otherwise, they are deemed not sustainable.

Table 1. Suggested Sustainability Indicators (Sis) for assessment in Construction stage by Construction Project Sustainability Assessing System (CPSAS) [24].

\begin{tabular}{cccc}
\hline SI & Abbreviation & Definition of Indicators & Criterion \\
\hline E1b1 & RBS & Ratio of Borrowed Soil & $\leq 50 \%$ \\
E1b2 & RCU & Ratio of Concrete Usage & $\leq 40 \%$ \\
E1c1 & MWS & Measure of Water Saving & $\geq 1$ \\
E1c2 & MWR & Measure of Water Recycle & $\geq 1$ \\
E1d1 & MES & Measure of Energy Saving & $\geq 1$ \\
E2a1 & APP & Measure of Air Pollution Prevention & $\geq 1$ \\
E2a2 & LAP & Usage of Low Air Pollution Method & $\geq 1$ \\
E2b1 & WPR & Measure of Water Pollution Reduction & $\geq 1$ \\
E2c1 & SWR & Measure of Solid Waste Reduction & $\geq 1$ \\
E2d1 & MNR & Measure of Noise Reduction & $\geq 1$ \\
E2e1 & AFT & Alternative for Toxicant & $\geq 1$ \\
E2e2 & GLP & Usage of Green Labeled Product & $\geq 10 \%$ \\
E2f1 & LGM & Low GHG Emission Method & $\geq 1$ \\
E3a1 & RPA & Ratio of Planting Area & $\geq 40 \%$ \\
E3a2 & EOH & Establishment of Habitation & Y \\
E3c1 & VGP & Usage of Vertical Green Planting & Y \\
S1a3 & CGB & Certified Green Building Items & $\geq 4$ \\
S1b1 & POD & Prevention of Disaster & Y \\
S1b2 & PSS & Protection of Stakeholders Safety & Y \\
S3a1 & FAD & Free Access for the Disabled & $\geq 1$ \\
S4a1 & PLR & Participation of Local Residents & Y \\
EC1a1 & RLE & Ratio of Local Employment & $\geq 20 \%$ \\
EC1a2 & SLR & Self-Liquidation Ratio & $\geq 50 \%$ \\
\hline
\end{tabular}

With the assessed CPSAS SIs, the overall Project Sustainability Index (PSI) can be calculated for a specific construction project. In Table 1, there are two types of indicators: (1) Quantitative indicators, as measured by the percentage (\%) values or quantities (No.) of the indicators; and, (2) Non-quantitative indicators, measured by 'Yes or $\mathrm{No}(\mathrm{Y} / \mathrm{N})^{\prime}$ ' regarding the outcome of the indicators. The two indicator types are aggregated in PSI using the following equation:

$$
P S I=\frac{\sum_{i=1}^{m} P S I_{n q}(i)+\sum_{j=1}^{n} P S I_{q}(j)}{m+n}
$$


where PSI is the Project Sustainability Index in percentage (\%), $m$ is the number of qualitative (non-quantitative) indicators, $\operatorname{PSI}_{n q}(i)$ is the evaluated result of the $i$ th qualitative indicator, $n$ is the number of quantitative indicators, and $\operatorname{PSI}_{q}(j)$ is the evaluated result of the $j$ th qualitative indicator.

By applying Equation (4), the obtained overall Project Sustainability Index (PSI) can be used as a measure to evaluate the 'Sustainability performance' of the project execution plan that was proposed by the bidder during the procurement process.

\section{Research Methodology and Procedure}

The research procedure adopted in the current research includes the following steps and methods:

(1) Construction of basic GAM-PEP graphs

The basic graphical model of the proposed GAM-PEP is developed using the Q-P Diagram that was originally proposed by Wang and Yu [13]. Different scenarios of heterogeneity in the Q-P Diagram are discussed and plotted to construct the three typical graphs for GAM-PEP, namely, G-I, G-II, and G-III.

\section{(2) Analysis of the heterogeneity of each GAM-PEP graph}

The heterogeneity of the three typical GAM-PEP graphs was analyzed to determine their 'market $\operatorname{PEP}\left(E_{m}\right)^{\prime}$ [12]. As the $E_{m}$ of either G-I or G-II is readily identifiable by plotting the tangents of all tender points on the Q-P Diagram, the associated $E_{m}$ can be easily estimated. However, the $E_{m}$ of Graph G-III is neither as sufficiently heterogeneous as G-I, nor apparently as homogeneous as G-II. As a result, it is further broken down to two sub-graph types, G-III(1) \& G-III(2), in order to estimate the associated $E_{m}$. To achieve this, each sub-graph of G-III is divided into sub-regions for detailed analysis.

(3) Selecting a more appropriate project procurement method

As the $E_{m}$ 's of Type G-I and Type G-II are readily identifiable, their associated more appropriate project procurement method can be easily determined. In order to select a more appropriate procurement method of Type G-III, the PEP $(E)$ of the sub-regions in G-III(1) \& G-III(2) are calculated while using a sub-quantitative method, where the overall $E_{m}$ 's of G-III(1) \& G-III(2) are obtained by adding up the estimated heterogeneities of all the sub-regions in these two sub-graphs to select a more appropriate procurement method.

\section{(4) Decision criteria of GAM-PEP}

Finally, four decision criteria to determine a more appropriate procurement method for a procurement project are summarized for the proposed GAM-PEP. Although the analysis is complex, the resulting decision criteria of GAM-PEP require no mathematical calculations, only the plotting of tender points on the Q-P Diagram. A more appropriate procurement method can then be determined by viewing the location and the direction of diagonal line of the graph type on the Q-P Diagram, i.e., G-I, G-II, G-III(1), and G-III(2).

(5) For application demonstration of the proposed GAM-PEP, a real-world building construction project was used as a case study to illustrate how a construction project emphasizing sustainability can be procured using the proposed GAM-PEP. Although there are several project-wise sustainability assessment frameworks available, some of them are post-execution assessment, while others lack a quantitative model for determining the project's sustainability score. No such frameworks are useful for construction project procurement conducted prior to project execution, and require quantitative measurement to efficiently estimate the sustainability performance of the bidder's project execution plan. In the case study, the Construction Project Sustainability Assessing System (CPSAS) [24] was adopted for measuring the sustainability performance of the project execution plan proposed by the bidder during the procurement stage, as CPSAS provides a useful pre-construction assessment and the Project Sustainability Index (PSI) to effectively determine the bidder's sustainability performance. 
The analysis results of GAM-PEP were also compared with those of the traditional P/PAM approach to show the improvement of GAM-PEP on decision making in selecting a more appropriate heterogeneous procurement method for sustainable construction projects.

\section{Development of Graphical Analysis Method for Price Elasticity of Performance (GAM-PEP)}

\subsection{Basic Graphs of GAM-PEP}

In the original Q-P Diagram (refer to Figure 1), the area between the LQ and the HQ and their relative heights are affected by the different characteristics of the procurement targets and the associated price elasticity of their performance. Such characteristics and the associated PEPs result in three types of Q-P Diagrams of the proposed GAM-PEP, denoted as G-I, G-II, and G-III, respectively, as shown in Figure 2.

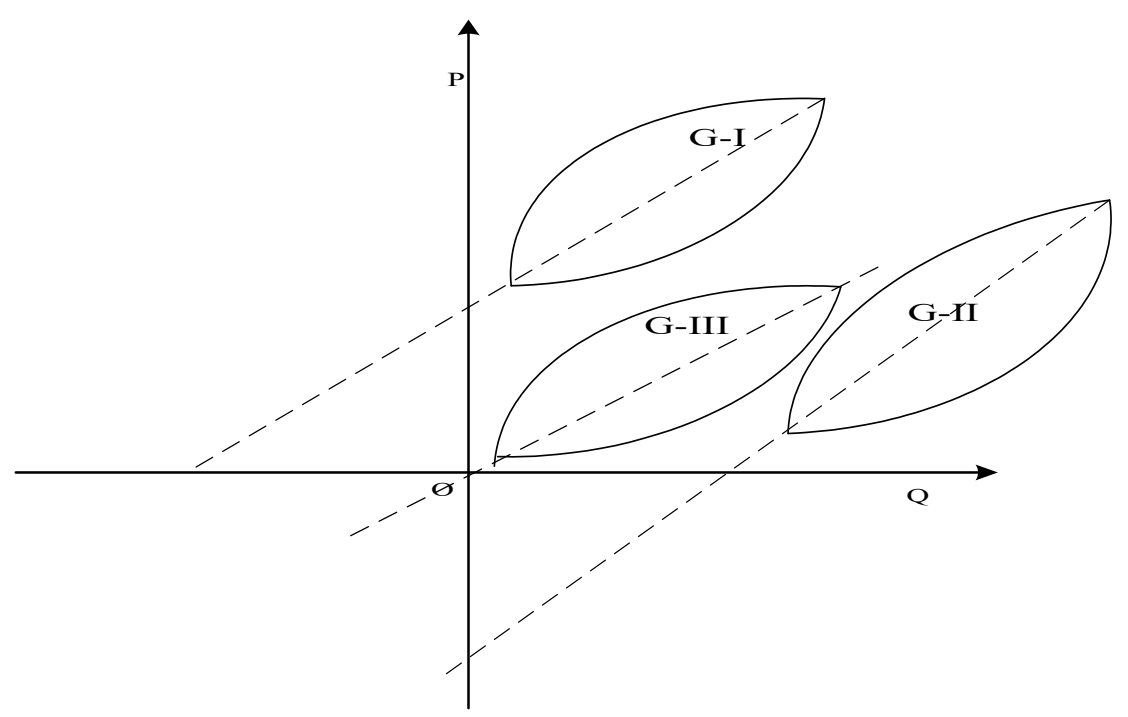

Figure 2. The typical Q-P graphs for Graphical Analysis Method for Price Elasticity of Performance (GAM-PEP).

\subsection{Estimating the Market Heterogeneity of GAM-PEP Graphs}

Using these three typical graphs, the procurement personnel can estimate the range for the market heterogeneity $\left(E_{m}\right)$ using the intersects of the tangent with the $X$-axis and the $Y$-axis. With the estimated $E_{m}$, the procurement personnel are able to determine which procurement method should be adopted. The following shows how to estimate the range of $E_{m}$ using Marshall's price elasticity theory; the three typical Q-P Diagrams are shown in Figure 2.

(1) Type G-I: a highly heterogeneous market

Type G-I is located in the upper-left area of the first quadrant. Thus, the tangents of points on G-I first intersect the $Y$-axis; the values for the PEP for the points are greater than 1 (for detailed analyses of PEP, please refer to Yu and Wang [12]).

(2) Type G-II: market with low heterogeneity

The Type G-II graph is located in the lower-right area of the first quadrant. The tangents first intersect the $X$-axis and then the $Y$-axis; thus, the PEP for Points are less than 1 (please refer to $Y u$ and Wang [12] for detailed analyses).

(3) Type G-III: moderately heterogeneous market 
When the Q-P Diagram resembles a Type G-III graph (see Figure 2), the analysis is more complicated than that of G-I and G-II. For a Type G-III graph, two tangents pass through (or very close to) the origin. These two tangents can be represented as ' $y=m x^{\prime}$. The tangents contact the HQ and LQ curves at Point-C and Point-D, respectively. The PEP for a Point-C on the HQ curve in Figure 3 is calculated using Equation (5).

$$
E_{C}=\frac{\frac{\Delta Q}{Q}}{\frac{\Delta P}{P}}=\left(\frac{\Delta Q}{\Delta P}\right) \times\left(\frac{P}{Q}\right)=\frac{1}{m} \times\left(\frac{P}{Q}\right)=\frac{Q}{P} \times\left(\frac{P}{Q}\right)=1
$$

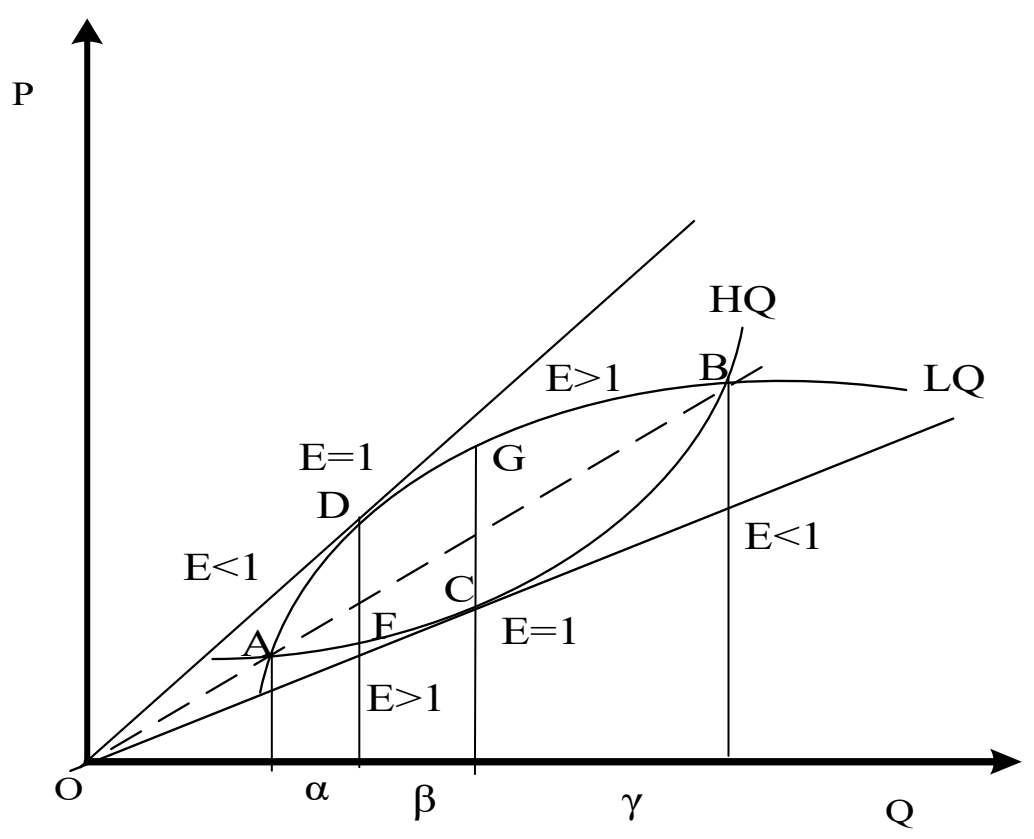

Figure 3. Type G-III graph (1).

Similar results can be obtained for $E_{D}$ by calculating the PEP of Point-D. The location of Point-C and Point-D on the $X$-axis (i.e., $x_{D}<x_{C}$ ) can be used to differentiate the two sub-graphs, as described in the following:

- Estimating the heterogeneity of Type G-III (1)

When $x_{D}<x_{C}$ (as shown in Figure 3), it is called Type G-III (1). For convenience in later analyses, the Type G-III (1) graph is divided into three sub-regions along the $X$-axis $(Q)$ : (1) $\alpha$, the sub-region to the left of Point-D, (2) $\beta$, the sub-region between Point-D and Point-C, and (3) $\gamma$, the sub-region to the right of Point-C.

The diagonal line (the dashed line) for Type G-III (1) usually intersects the $X$-axis and the $Y$-axis near the origin, $O$, which represents a moderate PEP procurement market with $E_{m} \fallingdotseq 1$. Further analysis is necessary to determine the appropriate project procurement method.

In Figure 3, four sub-regions (i.e., $A F$ and $F C$ of the $\mathrm{HQ}$ and $D G$ and $G B$ of the $\mathrm{LQ}$ ) are almost a heterogeneous procurement market, with $E>1$ and the other two sub-regions (i.e., $C B$ of the $H Q$ and $A D$ of the LQ) are almost a homogeneous procurement market, with $E<1$. The heterogeneity ( $E$ values) analyses of the sub-regions in Type G-III (1) are shown in Table 2.

Table 2. Heterogeneity analyses of Type G-III (1) (refer to Figure 3).

\begin{tabular}{cccc}
\hline Sub-Region & $\boldsymbol{\alpha}$ & $\boldsymbol{\beta}$ & $\boldsymbol{\gamma}$ \\
\hline$E$ values on the HQ & $E>1$ & $E>1$ & $E<1$ \\
$E$ values on the LQ & $E<1$ & $E>1$ & $E>1$ \\
\hline
\end{tabular}


- Estimating the heterogeneity of Type G-III (2)

When $x_{D}>x_{C}$ (as shown in Figure 4), it is called Type G-III (2). For convenience in later analyses, the Type G-III (2) graph is also divided into three sub-regions along the $X$-axis $(Q)$ : (1) $\alpha$, the sub-region to the left of Point-C, (2) $\beta$, the sub-region between Point-C and Point-D, and (3) $\gamma$, the sub-region to the right of Point-D. As shown in Figure 4, the diagonal line (the segmented line) for the Type G-III (2) also intersects the $X$-axis and the $Y$-axis near the origin, $O$, which represents a moderately heterogeneous procurement market with $E_{m} \fallingdotseq 1$, so further analysis is necessary to determine the appropriate contractor selection method.

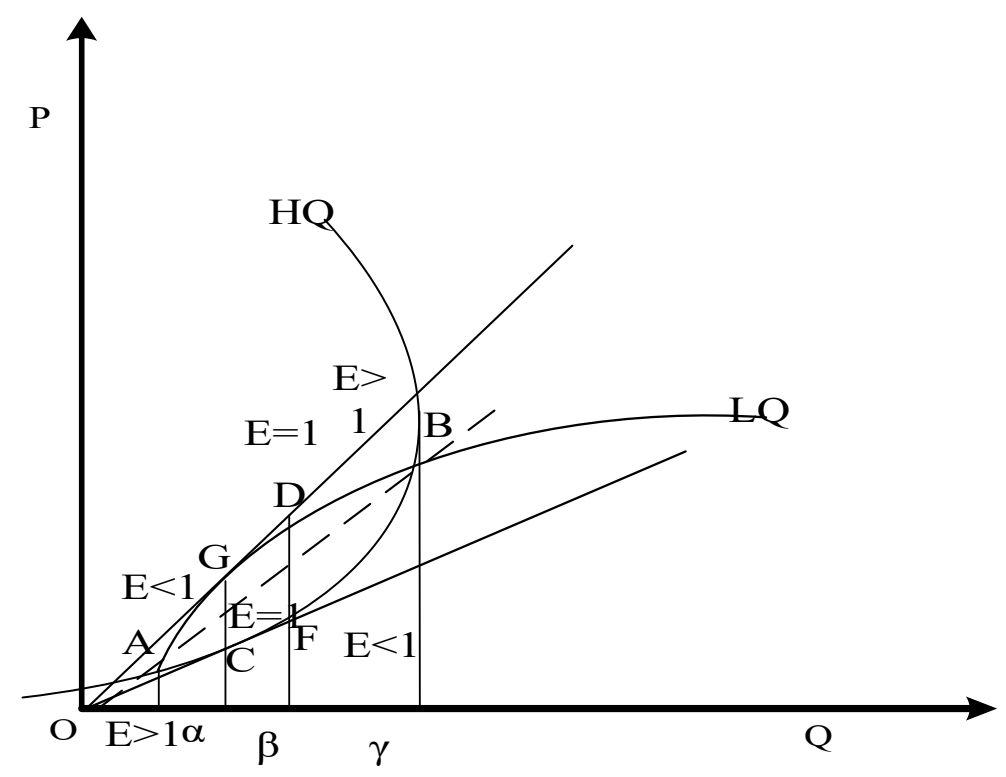

Figure 4. Type G-III graph (2).

In Figure 4, four sub-regions (i.e., $C F$ and $F B$ of the $\mathrm{HQ}$ and $A G$ and $G D$ of the $L Q$ ) are almost a homogeneous procurement market, with $E<1$; and, the other two sub-regions (i.e., $A C$ of the $\mathrm{HQ}$ and $D B$ of the LQ) are almost a heterogeneous procurement market, with $E>1$. The graphical analysis is shown in Table 3.

Table 3. Graphical analysis for Type G-III (2) (refer to Figure 4).

\begin{tabular}{cccc}
\hline Sub-Region & $\boldsymbol{\alpha}$ & $\boldsymbol{\beta}$ & $\boldsymbol{\gamma}$ \\
\hline$E$ values on the HQ & $E>1$ & $E<1$ & $E<1$ \\
\hline$E$ values on the LQ & $E<1$ & $E<1$ & $E>1$ \\
\hline
\end{tabular}

\subsection{Determination of a More Appropriate Project Procurement Method}

\section{(1) Type G-I \& Type G-II}

Based on the analyses of the three typical graphs for a GAM-PEP mentioned above, the procurement market for Type G-I is highly heterogeneous and BV is the most suitable method. Type G-II is highly homogeneous, so LT is the most suitable method.

(2) Type G-III graphs 
In order to analyze the heterogeneity of Type G-III, the overall value of $E_{m}$ for these three types of graphs must be calculated. The overall value for $E_{m}$ for Type G-III is the sum of the values for $E_{m}$ for all the sub-regions, $\alpha, \beta$, and $\gamma$, as calculated by Equation (6):

$$
E_{m}=E_{\alpha}+E_{\beta}+E_{\gamma}
$$

Using Equation (6) to calculate the value of $E_{m}$ for Type G-III (1) $\left(E_{m}^{I I I-(1)}\right)$ in Figure 3, results in Equation (7):

$$
\begin{aligned}
E_{m}^{I I I-(1)} & =\left(E_{m}^{D G}+E_{m}^{G B}+E_{m}^{A F}+E_{m}^{F C}\right)+\left(E_{m}^{A D}+E_{m}^{C B}\right) \\
& \cong 4(E>1)+2(E<1)
\end{aligned}
$$

Similarly, using Equation (6) to calculate the value for $E_{m}$ for Type G-III (2) $\left(E_{m}^{I I I-(2)}\right)$ in Figure 4, results in Equation (8):

$$
\begin{aligned}
E_{m}^{I I I-(2)} & =\left(E_{m}^{A G}+E_{m}^{G D}+E_{m}^{C F}+E_{m}^{F B}\right)+\left(E_{m}^{B D}+E_{m}^{A C}\right) \\
& \cong 4(E<1)+2(E>1)
\end{aligned}
$$

where $E_{m}^{I I I-(2)}$ is the overall market PEP for the procurement project; $E_{m}^{\prime A R C \prime}$ represents the PEP of arc ' $A R C^{\prime}$ ' and ' $A R C$ ' $=A G, G D, C F, F B, D B$, and $A C$.

Using Equations (7) and (8), ' $E_{m}^{I I I-(1)}-E_{m}^{I I I-(2)}$ ' is calculated to compare the value of $E_{m}$ for Types G-III (1) \& (2), as follows:

$$
\begin{gathered}
E_{m}^{I I I-(1)}-E_{m}^{I I I-(2)} \cong 2[(E>1)-(E<1)]>0 \\
\therefore E_{m}^{I I I-(1)}-E_{m}^{I I I-(2)}
\end{gathered}
$$

(3) Differentiation between Type G-III (1) and Type G-III (2)

The critical criteria for differentiating Type G-III graphs are the $\mathrm{X}$ coordinates of Point-C and Point-D. An alternative approach is to use the Cartesian X-Y coordinate system and let $E=1$ for Equation (1), yielding the tangent that passes through the origin, as in Equation (11):

$$
y=m x
$$

Using the second order quadratic function, $f(x)$, to model the HQ and LQ curves, as suggested by Yu and Wang [12], results in Equation (12).

$$
y=f(x)=a x^{2}+b x+c
$$

where $a, b$, and $c$ are constant coefficients.

Solving the following simultaneous equations gives $\mathrm{x}_{\mathrm{C}}$ :

$$
\left\{\begin{array}{c}
y_{1}=a_{1} x^{2}+b_{1} x+c_{1} \\
y_{1}=m_{1} x
\end{array} \Rightarrow C\left(x_{C}=\sqrt{\frac{c_{1}}{a_{1}}}, y_{C}=b_{1} \sqrt{\frac{c_{1}}{a_{1}}}+2 c_{1}\right)\right.
$$

where $a_{1}, b_{1}$, and $c_{1}$ are the constant coefficients for the quadratic equation of $y_{1}$.

Similarly, solving the following simultaneous equations for the LQ curve, $y_{2}$, and the tangent at Point-D gives $x_{D}$ :

$$
\left\{\begin{array}{c}
y_{2}=a_{2} x^{2}+b_{2} x+c_{2} \\
y_{2}=m_{2} x
\end{array} \Rightarrow D\left(x_{D}=\sqrt{\frac{c_{2}}{a_{2}}}, y_{D}=b_{2} \sqrt{\frac{c_{2}}{a_{2}}}+2 c_{2}\right)\right.
$$


With the coordinates $x_{C}$ and $x_{D}$, it is possible to determine if the procurement market belongs to Type G-III (1) or (2): if $x_{C}>x_{D}$, it belongs to Type G-III (1); otherwise, it belongs to Type G-III (2). In the proposed GAM-PEP, a simplified method to differentiate Type G-III (1) and Type G-III (2) is: (1) draw the straight line ' $y=m x^{\prime}$ connecting the two intersection points $\mathrm{A}$ and $\mathrm{B}$ of curves $\mathrm{HQ}$ and LQ; (2) draw tangent lines of HQ and LQ passing the origin point and locate the tangent points $C$ and D; (3) make judgements with the rule "if $x_{C}>x_{D}$, it belongs to Type G-III (1); otherwise, it belongs to Type G-III (2)".

\subsection{Summary of Decision Criteria}

Based on the above discussions, the decision criteria of GAM-PEP for a more appropriate project procurement method are summarized in Table 4.

Table 4. Summary of decision criteria for project procurement methods.

\begin{tabular}{ccc}
\hline Graph Type & Heterogeneity & Recommended Method \\
\hline Type G-I & High $\left(E_{m}>1\right)$ & BV \\
Type G-II & Low $\left(E_{m}<1\right)$ & LT \\
Type G-III (1) & Medium High $\left(x_{C}>x_{D}\right)$ & HLT $/$ BV \\
Type G-III (2) & Medium Low $\left(x_{C}<x_{D}\right)$ & HLT $/$ LT \\
\hline
\end{tabular}

\section{Demonstrated Applications}

In order to demonstrate the applicability of the GAM-PEP, a real-world building construction project was used as a case study. Two different project delivery models for the same project using the proposed GAM-PEP to select a more appropriate project procurement method were demonstrated. The case project involved a five-story building with the site area of $364 \mathrm{~m}^{2}$ and the floor area of $655 \mathrm{~m}^{2}$. The budget for the construction project was TWD 22,000,000 (USD 734,000). Project sustainability was emphasized by the project owner to be an important issue during project planning and procurement, as the project owner had implemented Corporate Social Responsibility (CSR) in her organization. Pursuing project sustainability in the project offered an extraordinary opportunity to show the project owner's commitment to CSR. The two delivery models were: (1) a Design/Build (D/B) model with design and construction works being awarded to the same contractor; and, (2) a traditional Design/Bid/Build (D/B/B) model: the project owner finishes the design work first, and then the construction work is awarded to a general contractor. In both project delivery models, the contractors were required to demonstrate their capability to implement sustainable project execution schemes by submitting a Project Sustainability Index (PSI) report according to their plan, to satisfy the requirements of the SIs defined in CPSAS [24].

In order to carry out the above-mentioned mission, an expert panel with five experienced members was organized by the project owner to perform the functionality of the Procurement Evaluation Committee (PEC): evaluating the performance of the submitted tenders. Nine local general contractors were identified and invited to bid for the project. The case study used two scenarios: (1) The D/B model, in which each bidder was required to acquire an architect and use a joint venture contract, to submit the construction plan with a design alternative for the residential project and according to the price for construction of the project; (2) The D/B/B model, in which each bidder was required to submit the construction plan and the price for the design plan provided by the project owner of the residential project. The application of the GAM-PEP for the two project delivery models is illustrated in the following two sub-sections.

\subsection{Case I: The D/B Model}

In the D/B model, five criteria were set by the PEC to evaluate the performance of the bidders for the D/B project: (1) Organization and experience (15\%), the qualifications of the professionals and previous experience of the bidder; (2) Project Sustainability Index (PSI) for Planning \& Design and 
Construction stages (25\%), a PSI assessment report on the overall project sustainability for Planning \& Design and Construction stages using the 27 SIs that were suggested by CPSAS; (3) Construction and quality control plan $(25 \%)$, the completeness and feasibility of the project management plan for construction work; (4) Schedule control (10\%), the rationality of the overall schedule and the feasibility progress control plan, and (5) Price (25\%), signifying the completeness and rationale of the bill of quantities (BOQ'B) for the project.

A total of nine contractors and the associated joint-venture architects submitted their plans and bid prices for the procurement project. The PEC then evaluated the submitted tenders. The tender evaluation results for the D/B model are shown in Table 5 . The definitions of symbols $m, k_{i}$, and $K_{i}$ are those for Equations (1)-(3).

Table 5. Bid evaluation results for the Design/Build (D/B) model.

\begin{tabular}{|c|c|c|c|c|c|c|c|c|c|c|}
\hline \multirow{2}{*}{ PEC Member } & \multicolumn{9}{|c|}{ Bidder } & \multirow{2}{*}{ Sum } \\
\hline & $\mathbf{B}_{1}$ & $\mathbf{B}_{2}$ & $\mathbf{B}_{3}$ & $\mathbf{B}_{4}$ & $\mathbf{B}_{5}$ & $\mathbf{B}_{6}$ & $\mathbf{B}_{7}$ & $\mathrm{~B}_{8}$ & B $_{9}$ & \\
\hline $\mathrm{A}$ & 71 & 65 & 72 & 80 & 84 & 90 & 88 & 75 & 91 & \multirow{6}{*}{3509} \\
\hline B & 65 & 63 & 67 & 77 & 78 & 88 & 85 & 69 & 88 & \\
\hline $\mathrm{C}$ & 70 & 67 & 70 & 82 & 83 & 92 & 89 & 74 & 92 & \\
\hline $\mathrm{D}$ & 68 & 65 & 73 & 82 & 85 & 91 & 90 & 73 & 90 & \\
\hline $\mathrm{E}$ & 61 & 60 & 68 & 79 & 80 & 88 & 83 & 69 & 89 & \\
\hline Sub & 335 & 320 & 350 & 400 & 410 & 449 & 435 & 360 & 450 & \\
\hline Ave. & 67 & 64 & 70 & 80 & 82 & 89.8 & 87 & 72 & 90 & \multirow{6}{*}{3.04} \\
\hline Price (1000) & 16,500 & 18,200 & 19,900 & 17,800 & 21,500 & 22,200 & 19,000 & 17,000 & 20,000 & \\
\hline$m$ & 6674 & 54,207 & 42,913 & 31,583 & 20,324 & 5641 & 44,996 & 16,254 & 50,744 & \\
\hline$E_{i}$ & 7.38 & 1.05 & 1.32 & 1.41 & 2.58 & 8.76 & 0.97 & 2.91 & 0.88 & \\
\hline$k_{i}$ & 0.10 & 0.09 & 0.10 & 0.11 & 0.12 & 0.13 & 0.12 & 0.10 & 0.13 & \\
\hline$k_{i} \times E_{i}$ & 0.70 & 0.10 & 0.13 & 0.16 & 0.30 & 1.12 & 0.12 & 0.30 & 0.11 & \\
\hline
\end{tabular}

(1) Analysis with traditional P/PAM

Using regression analyses, the HQ and LQ curves of the Q-P Diagram were respectively calculated using Equations (15) and (16):

$$
\begin{aligned}
& f(x)_{H Q}=191.61 x^{2}-121,705 x+40,000,000 \\
& R^{2}=0.9921 \\
& f(x)_{L Q}=-188.24 x^{2}+174,681 x-20,000,000 \\
& R^{2}=0.9943
\end{aligned}
$$

The procurement market for the D/B model is highly heterogeneous, because the Market Performance Elasticity of Price $\left(E_{m}\right)$ in Table 5 is $3.04 \geq 1.0$. As a result, the P/PAM analysis shows that the $\mathrm{BV}$ project procurement method is the best choice.

\section{(2) Analysis with the proposed GAM-PEP}

Using the GAM-PEP to analyze the D/B model, the GAM-PEP diagram was redrawn, as shown in Figure 5. The GAM-PEP diagram is relatively flat and the diagonal line intersects the $Y$-axis first and then the $X$-axis, revealing the graph type of G-I. As a result, the market is highly heterogeneous and the BV method is best suited for project procurement, which complies with the P/PAM analysis result. 


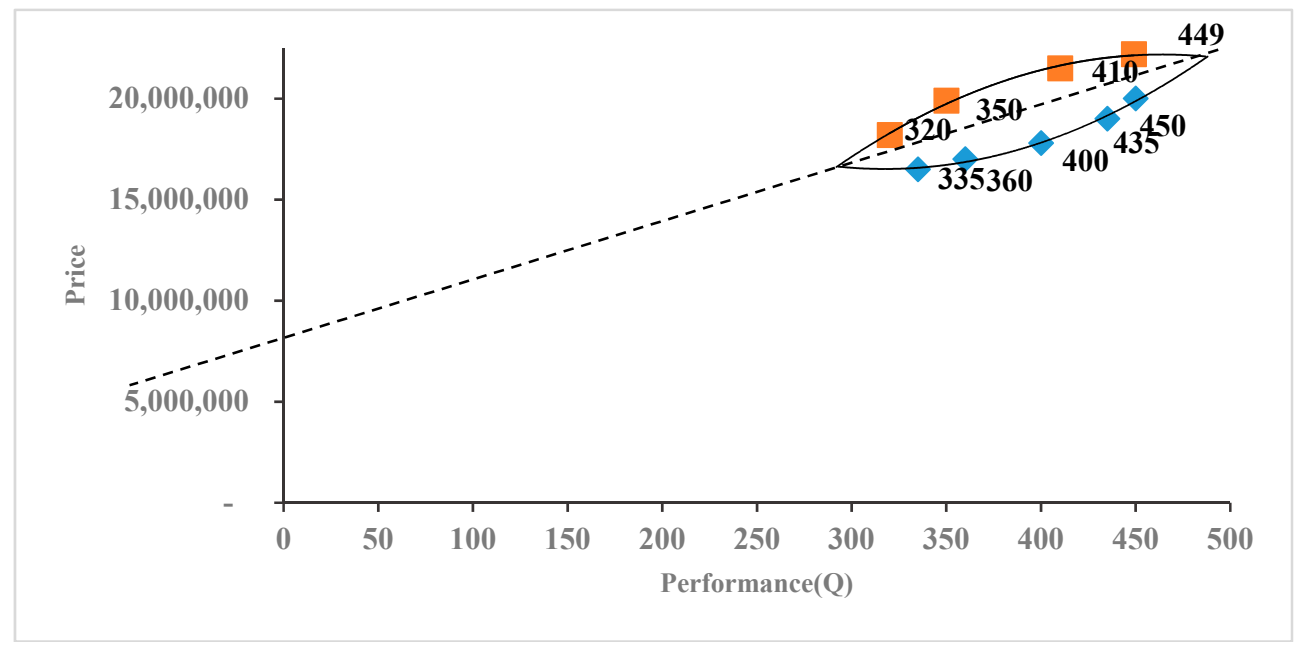

Figure 5. The GAM-PEP Diagram for the D/B model.

\subsection{Case II: The $D / B / B$ Model}

In Case II, the D/B/B model was adopted. The project owner initially acquired an architect to develop the design alternative and associated drawings. The design program and drawings were then provided as the appendix documents for the request for proposal (RFP). The nine invited bidders then submitted their construction plans (including the PSI reports) and the bid prices. As the design alternative was the same for all bidders, the evaluation criteria for the performance of the bidders were modified, as follows: (1) Organization and experience (20\%), the qualification of the professionals and previous experience of the bidder; (2) Project Sustainability Index (PSI) for the Construction stage $(20 \%)$, PSI assessment report on the overall project sustainability for Construction stage using the 23 SIs suggested by CPSAS; (3) Construction and quality control plan (30\%), the completeness and feasibility of the project management plan for construction works, interface integration, construction quality, material quality, and equipment quality; (4) Schedule control (10\%), the rationale of the overall schedule and the feasibility progress control plan; and, (5) Price (20\%), the completeness and rationale of the bill of quantities for the project.

The PEC evaluated the nine submitted tenders for the D/B/B model. The tender evaluation results are shown in Table 6.

Table 6. Tender evaluation results for the D/B/B model.

\begin{tabular}{|c|c|c|c|c|c|c|c|c|c|c|}
\hline \multirow{2}{*}{ PEC Member } & \multicolumn{9}{|c|}{ Bidder } & \multirow{2}{*}{ Sum } \\
\hline & $\mathbf{B}_{1}$ & $\mathbf{B}_{2}$ & $\mathbf{B}_{3}$ & $\mathbf{B}_{4}$ & $\mathbf{B}_{5}$ & $\mathrm{~B}_{6}$ & $\mathbf{B}_{7}$ & $\mathrm{~B}_{8}$ & $\mathrm{~B}_{9}$ & \\
\hline $\mathrm{A}$ & 75 & 71 & 78 & 79 & 86 & 87 & 84 & 80 & 86 & \multirow{6}{*}{3496} \\
\hline B & 71 & 66 & 75 & 75 & 81 & 85 & 81 & 78 & 83 & \\
\hline $\mathrm{C}$ & 72 & 68 & 74 & 76 & 80 & 83 & 82 & 74 & 84 & \\
\hline $\mathrm{D}$ & 69 & 65 & 72 & 71 & 79 & 82 & 78 & 73 & 82 & \\
\hline $\mathrm{E}$ & 73 & 70 & 79 & 74 & 84 & 86 & 83 & 75 & 87 & \\
\hline Sub & 360 & 340 & 378 & 375 & 410 & 423 & 408 & 380 & 422 & \\
\hline Ave. & 72 & 68 & 75.6 & 75 & 82 & 84.6 & 81.6 & 76 & 84.4 & \multirow{2}{*}{1.25} \\
\hline Price (1000) & 16,400 & 16,500 & 19,000 & 17,000 & 20,000 & 20,500 & 19,000 & 16,700 & 19,200 & \\
\hline
\end{tabular}

(1) Analysis with traditional P/PAM

Using the P/PAM analysis model, the HQ and LQ curves of the Q-P Diagram were respectively calculated using Equations (16) and (17):

$$
\begin{aligned}
& f(x)_{H Q}=202.11 x^{2}-107,522 x+30,000,000 \\
& R^{2}=0.9391
\end{aligned}
$$




$$
\begin{aligned}
& f(x)_{L Q}=-364.45 x^{2}+325,053 x-50,000,000 \\
& R^{2}=0.9967
\end{aligned}
$$

The procurement market for the model is moderately heterogeneous because the Market Performance Elasticity of Price $\left(E_{m}\right)$ in Table 6 is $1.25 \geq 1.0$. As a result, the P/PAM analysis shows that the $\mathrm{BV}$ project procurement method should be the most suitable choice.

(2) Analysis with the proposed GAM-PEP

Using GAM-PEP to analyze the procurement of the D/B/B model, the GAM-PEP Diagram was redrawn in Figure 6. Since the diagonal line intersects the Y- and X-axes near the origin in Figure 6, it is very similar to a Type G-III, so the values of $x_{C}$ and $x_{D}$ were calculated using Equations (12) and (13). It was found that $x_{C}=385$ and $x_{D}=370$ and $x_{C}>x_{D}$. The Q-P Diagram is Type G-III (1), but the market PEP, $E_{m}$, is only slightly greater than 1.0 , so the HLT method should be better suited for project procurement than $\mathrm{BV}$, which differs to that suggested by the traditional P/PAM method.

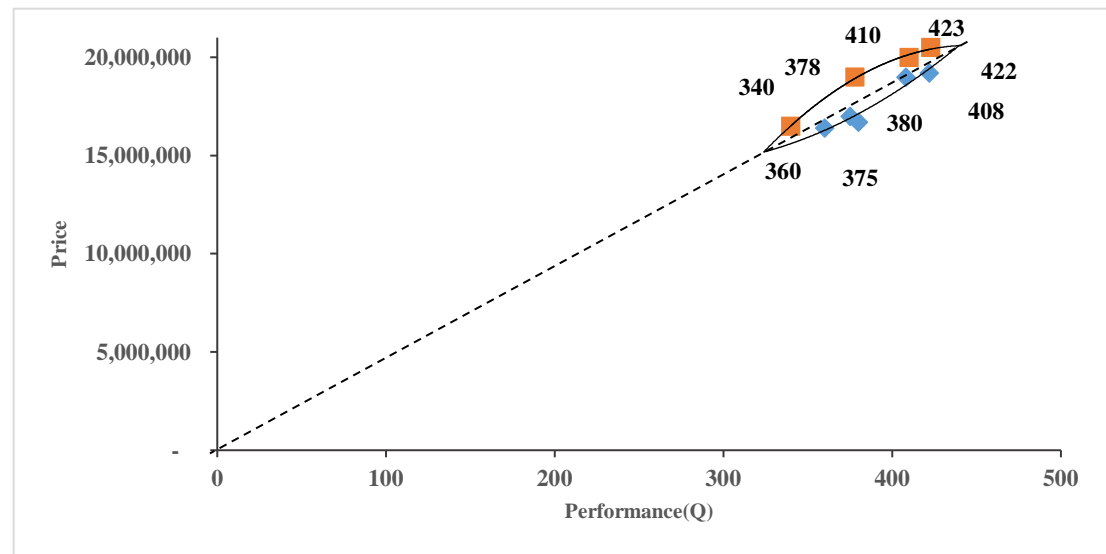

Figure 6. The GAM-PEP Diagram for the D/B/B model.

\section{Discussion}

\subsection{Comparison of the Case Study Results for the P/PAM and GAM-PEP}

From the results of the case study, the GAM-PEP recommends the BV method for the project procurement of a D/B construction project, the same as that of the P/PAM, since D/B projects are considered to be highly heterogeneous due to the different design alternatives and the project sustainability schemes proposed by the bidders. However, in the case of the D/B/B model, the GAM-PEP recommends the HLT method instead of BV, which differs from that of the P/PAM. A comparison of the case study results for the P/PAM and GAM-PEP is shown in Table 7.

Table 7. Comparison of the case study results for the P/PAM and GAM-PEP.

\begin{tabular}{ccc}
\hline \multirow{2}{*}{ Analysis Method } & \multicolumn{2}{c}{ Case } \\
\cline { 2 - 3 } & I. D/B Construction Project & II. D/B/B Construction Project \\
\hline \multirow{2}{*}{ P/PAM } & 3.04 & 1.25 \\
& BV & BV \\
\hline \multirow{2}{*}{ GAM-PEP } & G-I & G-III (1) \\
& BV & HLT \\
\hline
\end{tabular}

Moreover, the proposed GAM-PEP selects a more appropriate procurement method directly using graphs, without complicated mathematical calculations, which is much easier for the procurement personnel to implement when compared with traditional P/PAM. As a result, the proposed GAM-PEP 
fills the gap to improve the drawbacks of the traditional P/PAM method. This responds to the appeal of Yu and Wang [12] to find a better decision model for the procurement of heterogeneous projects.

\subsection{Advancement of the Knowledge Body in Project Procurement with GAM-PEP}

Table 7 shows that since the value of $E_{m}$ for Case II is approximately 1 , the traditional P/PAM method may fail to select a more appropriate project procurement method because with a moderate heterogeneity, as shown in this case, it must be pre-qualified by the PEC; then, the pre-qualified bidders will compete to reduce the procurement price. As a result, both delivery performance and cost-effectiveness can be pursued at the same time, in response to the requests of Abdelrahman et al. [6] and Wong et al. [8].

\subsection{Implication to Pursuing Project Sustainability}

In the demonstrated cases, project sustainability is considered in the project procurement stage by evaluating the PSI report for the submitted tenders, which are based on their proposals for the construction methods, energy reduction devices, environment-friendly products, etc., adopted in the project execution plan. With the combination of the proposed GAM-PEP and the previously developed CPSAS, a more sustainable project delivery approach can be realized. Such a systematic approach to attain project sustainability was not found in the relevant literature.

\section{Conclusions and Recommendations}

\subsection{Conclusions}

The procurement process significantly affects the successful delivery of a sustainable project. Project owners usually encounter market heterogeneity when selecting a more appropriate contractor for performing a project. This is especially important for the procurement of sustainable projects, since different bidders offer different schemes for achieving sustainability objectives. A decision support model that allows procurement personnel to make quality decisions in selecting a more appropriate procurement method is crucial. Previous models suffer from two drawbacks: (1) the requirement of more complicated mathematical calculations and a tedious process to determine market heterogeneity, and (2) the lack of criteria to determine the selection between BV and HLT when the tenders are moderately heterogeneous.

This study proposes a graphical analysis model, the GAM-PEP, to allow procurement personnel to select a more appropriate procurement method for a heterogeneous procurement project (including sustainable construction projects). From the case study results, it was found that the proposed GAM-PEP provides a simpler tool without the tedious mathematical calculations required in the traditional methods. Moreover, the proposed GAM-PEP also fills the gap of P/PAM in determining a project procurement method when the market is moderately heterogeneous. Such a model can be applied not only to construction projects but also to other types of procurement projects as long as the Q-P Diagrams can be constructed while using historical tendering data on specific project types.

The proposed GAM-PEP is especially useful for procuring sustainable projects, since the capability of achieving project sustainability by different contractors is usually heterogeneous. According to Yu et al. [19], a more sustainable project execution is usually also cost-effective. In such cases, a contractor is usually not selected using the traditional heterogeneous procurement method, unless HLT is adopted. With the combination of the proposed GAM-PEP and the previous CPSAS model, a more systematic approach to attain project sustainability is obtained.

\subsection{Recommendations}

Both traditional and GAM-PEP models require the price and performance data on the potential bidders in the market. Such information is difficult to acquire in practice. Although alternative 
approaches, such as using historical records or a market survey [13], were suggested, no systematic approach is available yet. A systematic method to collect data from the market is desired in the future.

This paper proposes a new decision-making model that considers the use of the HLT contractor selection method. However, the HLT is a relatively new option for project procurement, so further study is required to validate the application of HLT in real-world projects, especially for the heterogeneous sustainable projects. Such efforts deserve more future research efforts.

Author Contributions: For research articles with several authors, a short paragraph specifying their individual contributions must be provided. The following statements should be used "Conceptualization, K.-W.W. and W.-d.Y.; Methodology, K.-W.W. and W.-d.Y.; Software, Y.-Y.H.; Validation, Y.-Y.H. and S.-t.C.; Formal Analysis, Y.-Y.H.; Investigation, S.-t.C.; Resources, Y.-Y.H.; Data Curation, Y.-Y.H. and K.-W.W.; Writing-Original Draft Preparation, W.-d.Y.; Writing-Review \& Editing, W.-d.Y.; Visualization, W.-d.Y.; Supervision, S.-t.C.; Project Administration, W.-d.Y., please turn to the CRediT taxonomy for the term explanation. Authorship must be limited to those who have contributed substantially to the work reported.

Funding: This research received no external funding.

Conflicts of Interest: The authors declare no conflict of interest.

\section{References}

1. WTO. Agreement on Public Procurement. Government Procurement Agreement. World Trade Organization. Available online: https:/ /www.wto.org/english/docs_e/legal_e/gpr-94_e.pdf (accessed on 1 September 2018).

2. PCC. Government Procurement Act, Public Construction Commission. Available online: http://lawweb. pcc.gov.tw /EngLawContent.aspx?Type=E\&id=49\&KeyWord=Government+Procurement+Act (accessed on 1 September 2018).

3. Munasinghe, M. Environmental Economics and Sustainable Development; World Bank Publications: Washington, DC, USA, 1993; Available online: http:/ / documents.worldbank.org/curated/en/638101468740429035/pdf/ multi-page.pdf (accessed on 1 September 2018).

4. Hatush, Z.; Skitmore, M. Contractor selection using multi-criteria utility theory: An additive model. Build. Environ. 1998, 33, 105-115. [CrossRef]

5. Crowley, L.G.; Hancher, D.E. Evaluation of competitive bids. J. Constr. Eng. Manag. 1995, 121, $238-245$. [CrossRef]

6. Abdelrahman, M.; Zayed, T.; Elyamany, A. Best-Value Model Based on Project Specific Characteristics. J. Constr. Eng. Manag. 2008, 134, 179-188. [CrossRef]

7. Perng, Y.H.; Juan, Y.K.; Chien, S.F. Exploring the Bidding Situation for Economically Most Advantageous Tender Projects Using a Bidding Game. J. Constr. Eng. Manag. 2006, 132, 1037-1042. [CrossRef]

8. Wong, C.H.; Holt, G.D.; Cooper, P.A. Lowest price or value? Investigation of UK construction clients' tender selection process. Constr. Manag. Econ. 2000, 18, 767-774. [CrossRef]

9. Public Construction Commission. Submission Notes for HLT Procurement. PCC Official Letter, 2006.5.23 NOG: 09500191630; Public Construction Commission: Taipei, Taiwan, 2006.

10. Su, T.Y. The Research of the Lowest Bid Mechanism of Heterogeneous Procurement in Public Sectors-A Case Study of the Water Resources Agency, Ministry of Economic Affairs in Taiwan. Master's Thesis, Executive Master Program of Public Affairs, Tung Hai University, Taichung, Taiwan, June 2010. (In Chinese)

11. Chen, H.J. The Implementation Analysis of Awarding Principles in Government Procurement Act. Master's Thesis, Department of Civil Engineering, National Taiwan University, Taipei, Taiwan, June 2008. (In Chinese)

12. Yu, W.D.; Wang, K.W. Best Value or Lowest Bid? A Quantitative Elasticity Perspective. J. Constr. Eng. Manag. 2011, 138, 128-134. [CrossRef]

13. Wang, K.W.; Yu, W.D. Model for Analysis of Heterogeneity in Product Acquisition Procurement. J. Chin. Inst. Eng. 2011, 34, 877. [CrossRef]

14. Marshall, A. The Elasticity of Wants. In Principles of Economics—BOOK III; Macmillan and Co., Ltd.: London, UK, 1890; pp. 86-94.

15. Dolan, R.J.; Simon, H. Power Pricing-How Managing Price Transforms the Bottom Line; Free Press: New York, NY, USA, 1996. 
16. Labuschagne, C.; Brent, A.C. Sustainable Project Life Cycle Management: Aligning project management methodologies with the principles of sustainable development. In Proceedings of the 2004 PMSA International Conference, Johannesburg, South Africa, 10-12 May 2004; pp. 104-115.

17. Fernández-Sánchez, G.; Rodríguez-López, F. A methodology to identify sustainability indicators in construction project management-Application to infrastructure projects in Spain. Ecol. Indic. 2010, 10, 1193-1201. [CrossRef]

18. Shen, L.Y.; Tam, V.W.Y.; Tam, L.; Ji, Y.B. Project feasibility study: The key to successful implementation of sustainable and socially responsible construction management practice. J. Clean. Prod. 2010, 18, 254-259. [CrossRef]

19. Yu, W.D.; Cheng, S.T.; Miao, C.M.; Perng, G.Y. Green innovation of green roof technology-A case study. Mater. Sci. Eng. Technol. 2017, 48, 420-429.

20. Vijayaraghavan, K. Green roofs: A critical review on the role of components, benefits, limitations and trends. Renew. Sustain. Energy Rev. 2016, 57, 740-752. [CrossRef]

21. Lin, H.T. GHG emission reduction performance of state-of-the-art green buildings: Review of two case studies. Renew. Sustain. Energy Rev. 2016, 56, 484-493.

22. Mao, C.; Shen, Q.P.; Shen, L.Y.; Tang, L.N. Comparative study of greenhouse gas emissions between off-site prefabrication and conventional construction methods: Two case studies of residential projects. Energy Build. 2013, 66, 165-176. [CrossRef]

23. Huang, W.; Gao, Q.X.; Cao, G.L.; Ma, Z.Y.; Zang, W.D.; Chao, Q.C. Effect of urban symbiosis development in China on GHG emissions reduction. Adv. Clim. Chang. Res. 2016, 7, 247-252. [CrossRef]

24. Yu, W.D.; Cheng, S.T.; Ho, W.C.; Chang, Y.H. Measuring the Sustainability of Construction Projects throughout Lifecycle: A Taiwan Lesson. Sustainability 2018, 10, 1523. [CrossRef]

(C) 2018 by the authors. Licensee MDPI, Basel, Switzerland. This article is an open access article distributed under the terms and conditions of the Creative Commons Attribution (CC BY) license (http:/ / creativecommons.org/licenses/by/4.0/). 